\title{
Assessment of Mandibular Asymmetries in Adults: A Radiographic Study
}

Ahmad A Abdulmawjood BDS, MSc (Lec.)
Dept of Pedod, orthod, and Prev Dentistry

College of Dentistry, University of Mosul

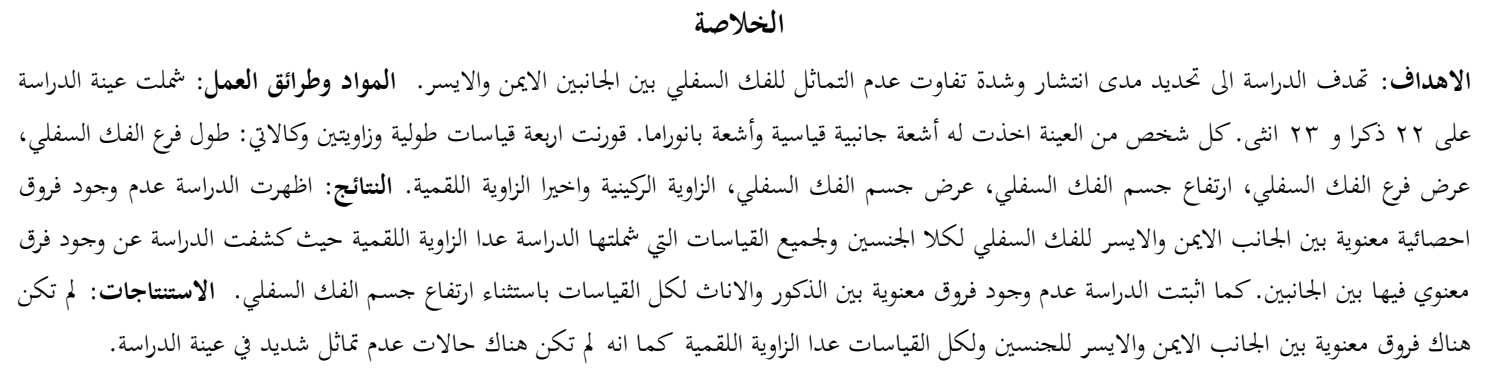

ABSTRACT

The mandibular asymmetry is important because of its direct effect on facial appearance. Aims: The aim of the present study was to determine the prevalence and severity of mandibular asymmetries between right and left sides. Materials and methods: The sample consisted of 22 males and 23 females having lateral cephalometric and orthopantomographic radiographs. Four linear and two angular measurements were compared between right and left sides of the mandible. These measurements were: ramus height $(\mathrm{RH})$, ramus width(RW), corpus height $(\mathrm{CH})$, corpus length(CL), gonial angle(Go) and condylar angle $(\mathrm{Co})$. Results: The study revealed no significant differences between right and left sides of the mandible, in both gender, regarding the 4 linear measurements. Whereas for the angular measurements there was a significant difference in condylar angle between right and left sides in both male and female groups. Also no significant gender differences were found regarding all measurements except in corpus height. Conclusion: No significant facial asymmetry was found between right and left sides of the mandible for the population being studied in both genders except in condylar angle. In addition it was found out that severe mandibular asymmetry is not so a common occurrence.

Abdulmawjood AA. Assessment of mandibular asymmetries in adults: A radiograghic study. $\mathrm{Al}-$ Rafidain Dent J. 2013; 13(2): 320-326.

Received: $15 / 4 / 2012$

Sent to Referees: $18 / 4 / 2012$ Accepted for Publication: 5/6/2012

\section{INTRODUCTION}

The word symmetry is derived from the Greek word symmetria which means 'of like measure'. Symmetry is defined as correspondence in size, shape and relative position of parts on opposite sides of a dividing line or median plane. Asymmetry is described as a lack or absence of symmetry. When applying this to the human face, it illustrates an imbalance or disproportionality between the right and left sides. ${ }^{(1)}$ The etiological factors of facial asymmetries and their mechanisms are not completely understood. ${ }^{(2)}$

Facial symmetry was first observed by the early Greek artists, and the term normal facial asymmetry was used. Later, Leonardo da Vinci and Albrecht Durer described the classic concept of human facial asymmetry and found absolute bilat- eral symmetry a normal morphologic characteristic. $^{(2)}$

Each individual shares many characteristics with the rest of the population but still unique in his or her own sense. This uniqueness is exhibited due to variation in size, shape and relationship of skeletal, dental and soft tissue facial structures. These variations may also exist within the same individual on the opposite sides of median sagittal plane of the face. ${ }^{(3)}$ The transverse dimension of the face affects the overall determination of the dentofacial proportions as well its balance and harmony. ${ }^{(4)}$

Morphological studies using radiography continue to provide useful results, and posteroanterior cephalograms play an important role in the evaluation of skeletal asymmetry. ${ }^{(5-7)}$ Submento-vertical radio- 
graph has also been proposed by Forsberg et $a{ }^{8)}$. to determine mandibular asymmetry. The panoramic radiograph offers a method to analyze the various structures of the mandible (e.g. CO, ramus, body) separately on the right and left sides. ${ }^{(9)}$

Since minor asymmetries of the human skeleton are common in the general population and usually have no esthetic or functional significance, ${ }^{(10)}$ therefore the aim of the present study was to use orthopantomograghic radiographs to assess the presence and severity of mandibular asymmetries in adults.

\section{MATERIALS AND METHODS}

The sample of the study consisted of 45 subjects, 22 males and 23 females, aged 18 to 30 years old. The sample was selected randomly from the patients attended the x-ray department of college of dentistry, Mosul University. Each subject had a lateral cephalometric and orthopantomographic (opg) radiographs. All radiographs were taken with the same digital cephalometric X-ray machine (Planmeca Dimax Pro. Finland). Only subjects showing class I skeletal pattern, according to Steiner analysis ${ }^{(11)}$ (SNA $82^{\circ} \pm 2^{\circ}$, SNB $78^{\circ} \pm$ $2^{\circ}$ ), in the lateral cephalometric radiograph were selected. In addition, only those panoramic radiographs presenting no artifacts, the whole mandible fully captured on the radiograph, and the contrast on the radiograph sufficient to perform all the intended measurements were chosen. ${ }^{(10)}$

For measurements, four linear and two angular measurements were taken to assess the asymmetry between left and right sides of the mandible.

The linear measurements are:

1. Ramus height (RH): perpendicular distance between the deepest point of the mandibular ramus notch (R1) and the lower border of the mandible (R2) as described by Ricketts. ${ }^{(12)}$

2. Ramus width (RW): perpendicular distance between the deepest point of the anterior border of the mandibular ramus (R3) and the posterior border of the ramus (R4) as described by Ricketts. ${ }^{(12)}$

3. Corpus height $(\mathrm{CH})$ : perpendicular distance between the lowest mesial point of the permanent first lower molar at the cemento-enamel junction (M1) and the lower border of the ramus (M2).

4. Corpus length $(\mathrm{CL})$ : distance between gonion intersection and pogonion $(\mathrm{Pg})$ in the midline, as recommended by Joondeph. ${ }^{(13)}$

The angular measurements included two angles:

1. Gonial (Go) angle was measured at the intersection of the planes formed by the posterior border of the mandibular ramus and the lower border of the mandibular corpus. ${ }^{(14)}$

2. condylar angle, which was measured by tracing a secant touching R1 on the ramus notch, running parallel to the Frankfort plane, and the long axis of the $\mathrm{CO}$ starting at condylion (C). ${ }^{(14)}$

To determine the distortion the panoramic radiograph may have caused on the linear dimensions of the mandible on both sides, a distortion factor for each hemimandible was calculated. For this, three radiographs from the sample were randomly selected. The mesio-distal length of the four permanent first molars was measured on the study models. The same distances were then measured on the panoramic radiographs. The distortion factor was calculated by dividing the mesiodistal length on the cast by the mesiodistal length on the radiograph for each of the four permanent first molars. ${ }^{(14)}$

The difference in vertical height between the two sides is expressed by an asymmetry index (AI), proposed by Sağlam, ${ }^{(15)}$ which is calculated with the formula $(\mathrm{R}-\mathrm{L}) /(\mathrm{R}+\mathrm{L}) \times 100 \%$.

The results were obtained as a percentage, where a positive result indicated that the right side was larger than the left, a negative result indicated that the left side was larger than the right, and a percentage equal to 0 indicated that both sides of the mandible were symmetric. Based on the asymmetry index (AI) for each measurement on each radiograph, the results were classified into four categories of asymmetry: no significant (NS) asymmetry, when $\mathrm{AI}$ was between 0 and 2.99 per cent; light (L), when $\mathrm{AI}$ was between 3 and 5 per cent; moderate $(M)$, when the index was greater than 5 per cent, but less than or equal to 10 per cent; and severe (S), when AI was more than 10 per cent. ${ }^{(14)}$ 
The difference between the right and left angle was used to determine the amount of asymmetry between the angles. The value of the left angle was subtracted from that of the right angle for both gonial and condylar angles. Thus, the severity of the asymmetry was determined as follows: $\mathrm{NS}$, when the difference between the right and left angle was between 0 and 2.99 degrees; L, when the difference between both sides was between 3 and 5 degrees; $\mathrm{M}$, when the difference was greater than 5 degrees but less than or equal to $10 \mathrm{de}-$ grees; and $\mathrm{S}$, when that difference was more than 10 degrees. ${ }^{(14)}$

The data were statistically analyzed using Wilcoxon paired analysis, a nonparametric test, at the 95 per cent level of confidence.

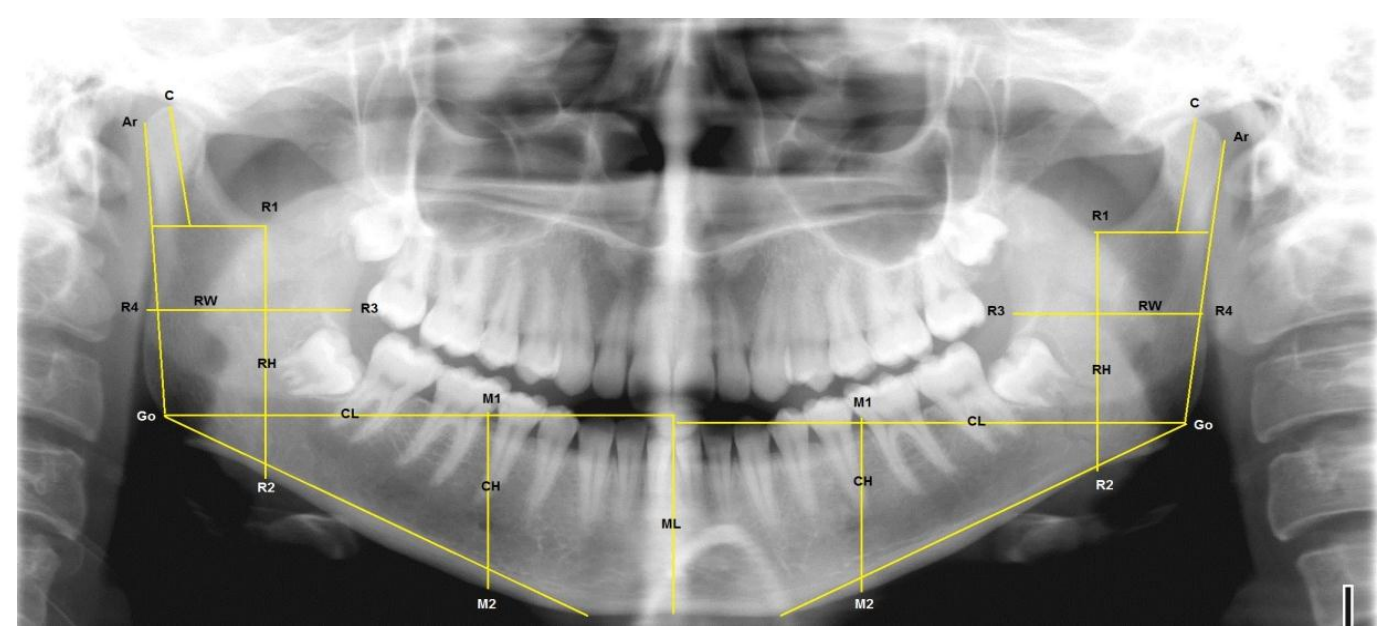

Figure (1) An orthopantomograph showing the landmark points and linear and angular measurements used in this study. (R1-R4) Points described by Ricketts ${ }^{(11)}$ at the mandibular ramus: Go, gonion; M1, point at the cervical point of the first permanent molar; M2, corresponding perpendicular point to M1 at the inferior border of the mandible; Ar, articulare; C, condylion; RW, ramus width; RH, ramus height; $\mathrm{CL}$, corpus length; $\mathrm{CH}$, corpus height; $\mathrm{ML}$, midline.

\section{RESULTS}

Tables (1) and (2) show descriptive statistics for the linear and angular measurements respectively, including minimum, maximum, mean and standard devi- ation. For all four longitudinal measurements, the means were higher on the right side compared with the left side for males and the opposite for females.

Table (1): Descriptive statistics for linear measurements.

\begin{tabular}{ccccccc}
\hline & measurements & N & Minimum & Maximum & Mean & Std. deviation \\
\hline \multirow{6}{*}{ Male } & CLr & 22 & 65.04 & 85.2 & 77.25 & 5.824 \\
& $\mathrm{CLl}$ & 22 & 63.91 & 86.1 & 76.04 & 6.076 \\
& $\mathrm{RWr}$ & 22 & 21.23 & 30.18 & 25.28 & 2.537 \\
& $\mathrm{RWl}$ & 22 & 20.65 & 29.89 & 25.13 & 2.382 \\
& $\mathrm{RHr}$ & 22 & 34.65 & 52.33 & 44.85 & 5.225 \\
& $\mathrm{RHl}$ & 22 & 34.3 & 55.09 & 44.23 & 5.246 \\
& $\mathrm{CHr}$ & 22 & 22.44 & 36.57 & 29.89 & 3.163 \\
\multirow{6}{*}{ Female } & $\mathrm{CHl}$ & 22 & 23.03 & 35.56 & 29.54 & 3.009 \\
\hline \multirow{6}{*}{} & $\mathrm{CLr}$ & 23 & 66.88 & 81.65 & 73.25 & 4.436 \\
& $\mathrm{CLl}$ & 23 & 63.91 & 82.25 & 74.32 & 5.409 \\
& $\mathrm{RWr}$ & 23 & 18.32 & 29.89 & 25.16 & 3.146 \\
& $\mathrm{RWl}$ & 23 & 19.81 & 32.62 & 25.91 & 3.331 \\
& $\mathrm{RHr}$ & 23 & 30.39 & 45.09 & 40.17 & 4.054 \\
& $\mathrm{RHl}$ & 23 & 31.22 & 44.8 & 39.74 & 3.929 \\
& $\mathrm{CHr}$ & 23 & 20.95 & 31.74 & 26.48 & 2.508 \\
& $\mathrm{CHl}$ & 23 & 20.72 & 32.41 & 27.07 & 2.964 \\
\hline
\end{tabular}

CL: corpus length, RW: ramus width, RH: ramus height, $\mathrm{CH}$ : corpus height, r: right and l: left. 
Table (2): Descriptive statistics for angular measurements.

\begin{tabular}{ccccccc}
\hline \multirow{5}{*}{ Male } & measurements & N & Minimum & Maximum & Mean & Std. deviation \\
\hline \multirow{4}{*}{ Female } & Gor & 22 & 111 & 127 & 119.31 & 3.846 \\
& Gol & 22 & 108 & 127 & 118.86 & 4.95 \\
& Cor & 22 & 90 & 108 & 99.13 & 4.733 \\
& Col & 22 & 90 & 107 & 191.68 & 4.684 \\
\hline & Gor & 23 & 114 & 134 & 120.34 & 5.201 \\
& Gol & 23 & 114 & 131 & 121.6 & 5.499 \\
& Cor & 23 & 90 & 110 & 98.82 & 3.845 \\
& Col & 23 & 94 & 102 & 102 & 4.482 \\
\hline
\end{tabular}

Go: gonial angle, Co: condylar angle, r: right and 1: left.

Wilcoxon paired analysis test for the data revealed no significant differences between right and left sides of the mandible, in males and females, regarding the four linear measurements as shown in Table (3).
Whereas for the angular measurements, shown in Table (4), the analysis revealed significant differences in condylar angle between right and left sides for both male and female groups. Meanwhile gonial angle showed no significant difference.

Table (3): Comparison of mandibular asymmetry between right and left sides' linear measurements.

\begin{tabular}{ccc}
\hline & Measurements & z-value \\
\hline \multirow{3}{*}{ Male } & CL & -1.57 \\
& RW & -0.4 \\
& RH & -1.93 \\
& CH & -1.73 \\
\hline \multirow{3}{*}{ Female } & CL & -1.18 \\
& RW & -1.34 \\
& RH & -1.27 \\
\hline
\end{tabular}

CL: corpus length, $\mathrm{RW}$ : ramus width, $\mathrm{RH}$ : ramus height, $\mathrm{CH}$ : corpus height.

Table (4): comparison of mandibular asymmetry between right and left sides' angular measurements.

\begin{tabular}{ccc}
\hline & Measurements & z-value \\
\hline Male & Go & -0.46 \\
Female & Co & $-3.33^{*}$ \\
\hline & Go & -1.6 \\
& Co & $-3.43^{*}$ \\
\hline
\end{tabular}

Go: gonial angle, Co: condylar angle

* Significant difference at $p \leq 0.05$

Concerning gender asymmetry difference, Wilcoxon analysis found out no significant statistical difference between males and females in the two angular measurements and linear measurements except in corpus height $(\mathrm{CH})$ as table (5) shows. 
Table (5): Comparison of mandibular asymmetry between males and females for linear and angular measurements.

\begin{tabular}{ccc}
\hline & Measurements & z-value \\
\hline \multirow{4}{*}{ Linear } & CL & 0.058 \\
& RW & 0.13 \\
& RH & 0.61 \\
\multirow{2}{*}{ Angular } & CH & $0.02^{*}$ \\
\hline & Go & 0.11 \\
& Co & 0.71 \\
\hline
\end{tabular}

CL: corpus length, RW: ramus width, $\mathrm{RH}$ : ramus height, $\mathrm{CH}$ : corpus height, Go: gonial angle, Co: condylar angle. * Significant difference at $p \leq 0.05$

The percentage of severity of mandibular asymmetry concerning linear and an- gular measurements are clarified in tables (6) and (7) respectively.

Table (6): Percentage for the severity of mandibular asymmetry of linear measurements.

\begin{tabular}{cclccc}
\hline \multirow{5}{*}{ Male } & \multicolumn{5}{c}{ Severity } \\
\cline { 2 - 6 } & Measurements & NS (\%) & L (\%) & M (\%) & S (\%) \\
\hline \multirow{3}{*}{ Female } & CL & 86.4 & 13.6 & - & - \\
& RW & 81.8 & 9.1 & 9.1 & - \\
& RH & 77.3 & 13.6 & 4.5 & 4.5 \\
& CH & 95.5 & 4.5 & - & - \\
& CL & 78.3 & 17.4 & 4.3 & - \\
& RW & 60.9 & 17.4 & 21.7 & - \\
& RH & 82.6 & 17.4 & - & - \\
\hline
\end{tabular}

CL: corpus length, RW: ramus width, RH: ramus height, $\mathrm{CH}$ : corpus height, NS: no significant asymmetry, L: light asymmetry, M: moderate asymmetry, S: severe asymmetry.

Table (7): Percentage for the severity of mandibular asymmetry of angular measurements.

\begin{tabular}{cccccc}
\hline & & \multicolumn{4}{c}{ Severity } \\
\hline & Measurements & NS (\%) & L (\%) & M (\%) & S (\%) \\
\hline \multirow{2}{*}{ Male } & Go & 36.36 & 36.36 & 27.3 & - \\
& Co & 45.5 & 27.3 & 27.3 & - \\
\hline \multirow{2}{*}{ Female } & Go & 34.8 & 52.2 & 13 & - \\
\hline & Co & 43.5 & 26.1 & 30.4 & - \\
\hline
\end{tabular}

Go: gonial angle, Co: condylar angle, NS: no significant asymmetry, L: light asymmetry, M: moderate asymmetry, S: severe asymmetry.

Regarding linear measurements, males had, in high percentages, light or no significant asymmetry differences between right and left sides for all linear measurements. $9.1 \%$ and $4.5 \%$ of males showed moderate asymmetry in ramus width (RW) and Height (RH) respectively. Only $4.5 \%$ of males had a severe asymmetry in ramus height (RH). Whereas in females, there was a lesser tendency for the "no significant asymmetry" and an increase in light and moderate asymmetry. No female experienced a severe asymmetry in linear measurement.
The percentages for asymmetry severity of the angular measurements showed that gonial angle presented percentages of $36.36 \%$ as "no significant asymmetry", $36.36 \%$ as light and $27.3 \%$ as moderate in males, while in females the percentage were as follows $34.8 \%$ for "no significant asymmetry", $52.2 \%$ for light asymmetry and $13 \%$ for moderate asymmetry. No severe asymmetry between right and left gonial angle were recorded in males and females. On the other, the percentages of asymmetry severity for the condylar angle were $45.5 \%$ for "no significant asym- 
metry", $27.3 \%$ for light asymmetry and $27.3 \%$ for moderate asymmetry in male group. While in females $43.5 \%$ of them showed "no significant asymmetry" and $26.1 \%$ and $30.4 \%$ had light and moderate asymmetry respectively. Again no severe asymmetry was recorded in condylar angle between left and right sides.

\section{DISCUSSION}

The present study showed that more than $90 \%$ of population tested had light or no significant asymmetry for all linear measurements used in the study. This finding contradict the study of Ramirez-Yañez et.al $^{(14)}$, focused on growing subjects, who found that more than half of the sample tested had moderate to severe, mandibular asymmetry, for the same 4 linear measurements, between right and left sides. This contradiction may be attributed to that young children may have functional displacement of the mandible that may disappear later on with the completion of permanent dentition or through orthodontic intervention.

In the current study, no significant differences, regarding all linear measurements, were found between right and left sides, a result which disagree with that of Ramirez-Yañez et.al ${ }^{(14)}$. On the other hand, this study revealed a statistical significant difference in condylar angle but not in gonial, whereas Ramirez-Yañez's et.al ${ }^{(14)}$ study found out statistical difference in both gonial and condylar angles. Similarly, the results of this study agree with those of Kurt et.al ${ }^{(16)}$ who also found no significant difference between right and left sides in concern with gonial angle.

Another interesting finding of the current study was the side predominance of mandibular asymmetries. While males showed right side predominance over left side, females showed the opposite i.e. left side predominance. This finding agrees with that of Kula et.al ${ }^{(17)}$ in concern with the male group and disagrees concerning females.

Another finding the present study was the absence of statistical difference between genders in angular and linear measurements except for corpus height which was statistically different. This finding is similar to that of Azevedo et.al ${ }^{(18)}$ and Ramirez-Yañez's et.al ${ }^{(14)}$.

\section{CONCLUSION}

The results of the present study revealed no significant facial asymmetry between right and left sides of the mandible for the population being studied in both gender. In addition, it was found that the presence of severe mandibular asymmetry is not so a common occurrence as far as the study's sample concern.

\section{REFERENCES}

1. Chia M SY, Naini FB and Gill DS. The aetiology, diagnosis and management of mandibular asymmetry. Orthodontic Update .2008; May: 44-52.

2. Menlik AK. A cephalometric study of mandibular asymmetry in a longitudinally followed sample of growing children. Am J Orthod Dentofac Orthop 1992;101:355-366.

3. Kumar N. An assessment of facial asymmetry in Chennai city population - A postero-anterior cephalometric study. Master thesis, 2005.

4. Basyouni AA. Clinical application forms for postero-anterior cephalometric analysis. The Saudi Dental J; 1997;9(2): 66-77.

5. Pirttiniemi PM. Associations of mandibular and facial asymmetries: A review. Am J Orthod Dentofacial Orthop. 1994; 106: 191-200.

6. Haraguchi S, Takada K, Yasuda Y. Facial asymmetry in subjects with skeletal Class III deformity. Angle Orthod. 2002;72:28-35.

7. Janson GR, Metaxas A, Woodside DG, de Freitas MR, Pinzan A.Threedimensional evaluation of skeletal and dental asymmetries in Class II subdivi sion malocclusions. Am J Orthod Dentofacial Orthop. 2001; 119:406-418.

8. Forsberg C T, Burstone C J, Hanley K J. Diagnosis and treatment planning of skeletal asymmetry with the submental-vertical radiograph. American J Orthodontics. 1984; 85: 224-237.

9. Larheim T A, Svanaes D B . Reproducibility of rotational panoramic radiography: mandibular linear dimensions 
and angles. Am J Orthod Dentofacial Orthop 1986; 90: 45-51

10. Skolnick J, Iranpour B, Westesson PR, Adair S. Prepubertal trauma and mandibular asymmetry in orthognathic surgery and orthodontic patients. Am J Orthod Dentofacial Orthop. 1994; 105: 73-77.

11. Proffit WR. Contemporary Orthodontics, $4^{\text {th }}$ ed. St Louis: MosbyYearbook, 2007:208

12. Ricketts R M. Cephalometric analysis and synthesis. Angle Orthodontist 1961; 31: 141-156.

13. Joondeph DR. Mysteries of asymme tries. Am J Orthod Dentofacial Orthop. 2000; 117: 577-579.

14. Ramirez-Yañez GO, Stewart A, Franken E and Campos K. Prevalence of mandibular asymmetries in growing patients. European Journal of Ortho- dontics 2010; August 19:1-7

15. Saglam A M. The condylar asymmetry measurements in different skeletal patterns. J Oral Rehabilitation. 2003; 30: 738-742.

16. Kurt G, Uysal T, Sisman Y, Ramoglu S I. Mandibular asymmetry in Class II subdivision malocclusion. Angle Orthodontist. 2008; 78: 32-37

17. Kula K A, Esmailnejad A, Hass A Dental arch asymmetry in children with large overjets. Angle Orthodontist. 1998; 68: 45-52.

18. Azevedo A R, Janson G, Henriques $\mathbf{J}$ F, Freitas M R. Evaluation of asymmetries between subjects with Class II subdivision and apparent facial asymmetry and those with normal occlusion. Am J Orthod Dentofacial Orthop. 2006; 129: 376-383. 